\title{
RED FRACTION AMONG SATELLITE GALAXIES WITH DISK-LIKE LIGHT PROFILES: EVIDENCE FOR INFLOW IN THE H I DISK
}

\author{
J. A. Hester \\ California Institute of Technology, Pasadena, CA 91125, USA; jhester@srl.caltech.edu \\ Received 2007 April 25; accepted 2010 July 7; published 2010 August 6
}

\begin{abstract}
The relationships between color, characterized with respect to the $g-r$ red sequence; stellar structure, as determined using the $i$-band Sérsic index; and group membership are explored using the Sloan Digital Sky Survey (SDSS). The new results place novel constraints on theories of galaxy evolution, despite the strong correlation between color and stellar structure. Observed correlations are of three independent types- those based on stellar structure, on the color of disk-like galaxies, and on the color of elliptical galaxies. Of particular note, the fraction of galaxies residing on the red sequence measured among galaxies with disk-like light profiles is enhanced for satellite galaxies compared to central galaxies. This fraction increases with group mass. When these new results are considered, theoretical treatments of galaxy evolution that adopt a gas accretion model centered on the hot galactic halo cannot consistently account for all observations of disk galaxies. The hypothesis is advanced that inflow within the extended H I disk prolongs star formation in satellite galaxies. When combined with partial ram pressure stripping (RPS) of this disk, this new scenario is consistent with the observations. This is demonstrated by applying an analytical model of RPS of the extended Hi disk to the SDSS groups. These results motivate incorporating more complex modes of gas accretion into models of galaxy evolution, including cold mode accretion, an improved treatment of gas dynamics within disks, and disk stripping.
\end{abstract}

Key words: galaxies: clusters: general - galaxies: evolution

Online-only material: color figures

\section{INTRODUCTION}

Galaxies in clusters have earlier type morphologies, redder colors, and lower star formation rates (SFRs) than galaxies in a field of similar luminosity (Dressler 1980; Gomez et al. 2003; Goto et al. 2003). Galaxies in groups and clusters formed earlier and in denser environments than their field counterparts. Cluster galaxies may therefore naturally appear older than field galaxies. In addition, group and cluster galaxies are more likely to have undergone major mergers (Göttlober et al. 2001) and are susceptible to harassment (Moore et al. 1998), both of which may transform blue, late-type galaxies into red, earlytype galaxies. Furthermore, galaxies in groups and clusters no longer accrete fresh gas and their galactic halo gas is easily heated and stripped, together termed "strangulation" (Larson et al. 1980; Balogh et al. 2000). They are also subject to ram pressure striping (RPS) of their gas disks (Gunn \& Gott 1972). These interactions with the intergalactic medium (IGM) are capable of suppressing star formation in group and cluster members, resulting in redder colors, but do not alter their structural morphology.

Observations indicate that some combination of the physical processes that act in groups and clusters is responsible for the bulk of the evolution of the full galaxy population. Earlytype galaxies with red colors and concentrated stellar profiles are found preferentially in dense environments (Hogg et al. 2003; Kauffmann et al. 2004; Blanton et al. 2005a) and are more clustered than late-type galaxies (Norberg et al. 2002; $\mathrm{Li}$ et al. 2006). Previous studies using group catalogs in the SDSS and 2dFGRS have found that the fraction of passive red galaxies increases with group mass (Martínez et al. 2002; Weinmann et al. 2006a; Hester 2006b), though other studies have disagreed with this conclusion (Balogh et al. 2004; Tanaka et al. 2004; van den Bosch et al. 2008). (Note that Hester $2006 \mathrm{~b}$ is a precursor to the current work.) These related observations can all be tied to group and cluster scales. The morphology-density relation on intermediate and large scales is reducible to correlations on scales of 1-2 Mpc, similar to the virial radius of a cluster (Blanton et al. 2006b). Furthermore, the enhanced early-type fraction in groups and clusters gives rise to amplified clustering beyond 1-2 Mpc (Collister \& Lahav 2005; Blanton et al. 2007). Massive halos cluster strongly and are found in dense environments and their satellites share these properties. Moreover, observations made at intermediate redshifts indicate that significant galaxy evolution occurs in group-like environments (Cooper 2006, 2007; Gerke 2007).

Analyses of large galaxy redshift surveys have revealed strong bimodalities in both stellar properties; color, SFR, 4000 A break; and structural properties; concentration, $i$-band Sérsic index $\left(n_{i}\right)$, central surface brightness ( $\mu$; Strateva et al. 2001; Blanton et al. 2003b; Kauffmann et al. 2003a; Baldry et al. 2004; Li et al. 2006). Hence, galaxies can be classified neatly as either earlytype or late-type based on either their colors or light profiles.

The correlation between indicators of star formation history (SFH) and environment is stronger than that between measures of stellar structure and environment (Kauffmann et al. 2003b). This is reflected in the relative amplitudes of the correlation function of galaxies that are red, have large $4000 \AA$ breaks, high concentrations, or bright $\mu$. All are enhanced compared to the full galaxy population, but galaxies with red colors or a large $4000 \AA$ break show a greater enhancement ( $\mathrm{Li}$ et al. 2006). This observation reflects a fundamental asymmetry between SFH and structure. At fixed $g-r$ color, the distribution of $n_{i}$ is independent of environment, but at fixed $n_{i}$ the distribution of $g-r$ color is sensitive to environment (Quintero et al. 2006). Hence, color is a better predictor of environment (Blanton et al. 2005a; see also van den Bosch et al. 2008). This asymmetry indicates that at least one process frequently occurs in grouplike environments that affects star formation, but not structure. Strangulation and RPS are two such processes. 
In order to constrain the impact of the physical processes that occur in groups and clusters, in particular RPS and strangulation, this paper explores the relationship between the $\mathrm{SFH}$, measured with respect to the $g-r$ red sequence; stellar structure, as measured by $n_{i}$, and environment, characterized by group membership, in more detail than has been done previously. This analysis must take into account known correlations between SFH, stellar structure, luminosity, and environment. Galaxies show a strong tendency to be either blue with disk-like light profiles (low Sérsic index and central surface brightness), or red with elliptical-like light profiles (high Sérsic indexes and central surface brightness; Blanton et al. 2005a). Both color and structure correlate strongly with luminosity and stellar mass, tending toward earlier types with increasing luminosity or mass (Madgwick et al. 2002; Kauffmann et al. 2003b; Baldry et al. 2004; Balogh et al. 2004; Blanton et al. 2005a; Weinmann et al. 2006a; Kelm et al. 2005). The luminosity function correlates with environment, tending toward brighter galaxies in denser environments or more massive groups/clusters (Yang et al. 2003, 2005; Croton et al. 2005; Zheng et al. 2005). The joint correlations between galaxy-type and luminosity, and luminosity and environment are of particular importance to the study presented here. It is essential to separate galaxies by luminosity and group mass simultaneously, or to attempt to correct for these correlations. I chose the first approach.

To date, models of the evolution of galaxy populations, which are generally semi-analytic models (SAMs), rely on strangulation, essentially the stripping of the galactic halo, to drive galaxies onto the red sequence after they are accreted by a group or cluster (e.g., Bower et al. 2006; Croton et al. 2006). In these models all satellite galaxies that were star forming at the time of their accretion transition onto the red sequence over 1-2 Gyr as they consume the gas within their stellar disks. These SAMs, which I will refer to as conventional SAMs, were initially successful at reproducing the properties of observed galaxy populations but failed when confronted with observations of the fraction of satellite galaxies that have blue colors (Weinmann et al. 2006b). The observed blue fraction is higher than predicted by conventional SAMs and decreases as the mass of the host group increases, which is not seen in the models.

A new iteration of semi-analytic modeling invokes partial stripping, as opposed to complete stripping, of the galactic halo to address this conflict (Font et al. 2008; Kang \& van den Bosch 2008). In recent hydrodynamical simulations, the galactic halo is only partially stripped in groups and the efficiency of galactic halo stripping decreases with group mass (McCarthy et al. 2008). Unstripped galactic halo cores have been observed around elliptical galaxies in clusters, in agreement with the simulation results (Sun et al. 2007). Incorporating partial stripping alleviates the conflict with observations. Partially stripped satellites continue to form stars for longer, which increases the overall fraction of star-forming satellites, and the efficiency of partial stripping increases with group mass, effectively shortening the typical star-forming life of a satellite and decreasing the blue fraction.

While introducing partial galactic halo stripping appears to fix the SAMs, the assumed physics may not be a valid description of real galaxies. These models assume accreted gas is shock-heated at the virial radius, forming a galactic halo around both spiral and elliptical galaxies. Upper limits have been placed on the X-ray luminosities of spiral galaxy galactic halos which are inconsistent with this simple picture of gas accretion (Benson et al. 2000; Rasmussen et al. 2009). Recent models of gas accretion onto galaxies highlight the contribution of cold flows, gas which reaches the inner regions of the dark matter halo without shocking or contributing to the hot galactic halo (Birnboim \& Dekel 2003; Kereš et al. 2005; Dekel \& Birnbiom 2006). These models predict that spiral galaxies have galactic halos with lower densities than in SAMs and hence lower X-ray luminosities.

The stripping simulations of McCarthy et al. (2008) which are the basis for the new SAMs assume a relatively dense galactic halo, such as is observed around elliptical galaxies. Hester (2006a) presents an analytical model of RPS of both a gas disk and its associated hot galactic halo. This model reproduces both simulations and observations where available, and in it the assumed density of the galactic halo is comparable to the upper limits set by the X-ray observations of late-type galaxies. Benson et al. (2000) place upper limits of $L_{x}=0.4$ and $1.2 \times 10^{41} \mathrm{erg} \mathrm{s}^{-1}$ on two galaxies with estimated virial temperatures of 0.232 and $0.368 \mathrm{keV}$, respectively. The model $L_{x}$ for these virial temperatures are 0.7 and $1.4 \times 10^{41} \mathrm{erg} \mathrm{s}^{-1}$, corresponding to 0.6 and $1.5 \times 10^{10} M_{\odot}$ of gas in the galactic halo. The model is also consistent with the similar observational limits of Rasmussen et al. (2009). Despite accepting the upper limits on the galactic halo density, Hester (2006a) found that galactic halo stripping is extremely efficient for spiral galaxies (see Figure 4 of the present work).

The high efficiency of galactic halo stripping in disk satellites presents a significant challenge to SAMs that postulate that inefficient stripping of this halo accounts for the frequency of blue satellites. The majority of blue, star-forming galaxies are disks. Red disks are known to exist in dense environments and contribute to the strong correlation between color and environment. It is therefore likely that the excess, compared to conventional SAM predictions (e.g., Weinmann et al. 2006b), of blue satellite galaxies is composed of disk galaxies. If this is so then it is unlikely that partial stripping of the hot galactic halo can reconcile SAMs with the observations, particularly if the fraction of red disk galaxies depends on group mass.

If partial galactic halo stripping does not account for the discrepancy between models and observations, then a new physical mechanism must be advanced in its place. A disk of neutral atomic gas is observed extending beyond the stellar disk of most spiral galaxies. Milky Way (MW)-like galaxies in groups are partially ram pressure (RP) stripped of this extended gas disk (Hester 2006a). If one postulates sufficient inflow of gas within this disk, then the unstripped portion of the $\mathrm{H}_{\mathrm{I}}$ disk can support star formation after the satellite is accreted. This would allow satellites to continue to form stars for longer than in conventional SAMs, thereby increasing the fraction of satellite galaxies that are currently forming stars. The efficiency of RPS increases with group mass, which can account for the observed dependence of the red fraction on group mass. Inflow within the H I disk plus partial RPS of this disk is an analog of partial galactic halo stripping which occurs explicitly in disk satellites.

To distinguish between modes of gas accretion and more generally introduce further constraints on models of galaxy evolution, new observational results are presented here that explore the correlations between color, structure, luminosity, and environment in more detail than has been done previously. This work uses the catalog of SDSS groups presented in Berlind et al. (2006). The catalog is reviewed briefly in Section 2. The new observational results are presented and discussed in Section 3. In Section 4, I further motivate the disk inflow plus 
RPS scenario and demonstrate that partial RPS occurs in the SDSS group catalog. Section 5 concludes.

\section{THE GROUP CATALOG}

The Sloan Digital Sky Survey (SDSS; York et al. 2000) conducted an imaging and photometric survey of $\pi$ sr in the northern hemisphere as well as three thin slices in the southern hemisphere and obtained optical spectra of some 930,000 galaxies. Observing was done using a dedicated $2.5 \mathrm{~m}$ telescope in Apache Point, New Mexico. The telescope operated in drift scan mode and observed in five bandpasses (Fukugita et al. 1996). Magnitude calibration was carried out using a network of standard stars (Smith et al. 2002). Three sets of spectroscopic targets were selected automatically, the main galaxy sample, the luminous red galaxy sample, and the quasar sample (Eisenstein et al. 2001; Richards et al. 2002; Strauss et al. 2002). Objects in the main galaxy sample have Petrosian magnitudes $r^{\prime}<17.7$ and are classified as extended. Magnitudes were corrected for galactic extinction using the reddening maps of Schlegel et al. (1998) prior to selection. Spectroscopy was taken using a pair of fiber-fed spectrographs, and targets were assigned to fibers using an adaptive tiling algorithm (Blanton et al. 2003a). Data reduction for the SDSS was done using a series of automated pipelines (Hogg et al. 2001; Smith et al. 2002; Pier et al. 2003; Ivezić et al. 2004; Lupton et al. 2001).

The group catalog was assembled from a volume-limited sample drawn from the large-scale structure sample, sample 14 of the New York University Value Added Galaxy Catalog (NYU-VAGC; Blanton et al. 2005c). The sample is based on SDSS Data Release 3. The sample goes down to $M_{r}<-19.0$ and has a redshift range of $0.015-0.068$. The group finding algorithm is described in detail in Berlind et al. (2006). The group finder is a friends of friends algorithm with two linking lengths, one for projected distances and one in redshift. Linking lengths are chosen such that the multiplicity function, richness, and projected size of groups recovered from simulations that were projected into redshift space are unbiased measures. The linking lengths are also chosen to maximize the number of groups recovered and minimize the number of spurious groups. Fiber collisions are treated by assigning each "collided" galaxy the redshift of its nearest neighbor. The velocity dispersions of the recovered groups are systematically low because the group finder does not link the fastest moving group members to the group.

Virial masses and radii for the groups are determined by assuming a monotonic relationship between group mass and total group luminosity and then matching a $\Lambda \mathrm{CDM}$ mass function to the measured group luminosity function (Berlind et al. 2006). The group finder is tuned to recover the multiplicity function of groups, and should therefore also recover the group luminosity function. This matching assumes no scatter in the relationship between mass and richness. Individual mass measurements are therefore noisy. In this paper, galaxies are placed into wide bins in group mass, each of which contains hundreds of groups. Groups whose measured mass places them in the appropriate mass bin do not affect the results presented here. Scattering of groups between the bins will, however, reduce the effective difference in the group mass between bins thereby biasing against observing other differences across the group mass bins. Group mass and group luminosity are correlated, however, and the large number of groups in the catalog should ensure significant differences in the distribution of $M_{\mathrm{gr}}$ between the bins.
Both RPS and strangulation only affect the satellite galaxies within a group or cluster. Therefore, the catalog is split into "satellite" galaxies and "central" galaxies. This was done in two ways. First, for groups with more than one member, "central" galaxies were selected by requiring that a central galaxy have an absolute $r$-band magnitude, $M_{r}$, at least 0.5 brighter than the next brightest galaxy in the group. Thus, central galaxies were conservatively selected and not all groups were required to have a central galaxy. All galaxies that were not assigned to the sample of central galaxies were assigned to the satellite sample. Second, a distance criteria was introduced and all groups were required to have a central galaxy. A galaxy which was $0.5 M_{r}$ brighter than the next brightest galaxy was still selected as the central galaxy. When no such dominant galaxy was identified, among galaxies with $M_{r}$ within 0.5 of the luminosity of the brightest galaxy the galaxy nearest the group center was selected. In both cases "groups" with only one member were assigned to the central galaxy sample, and pairs, groups with only two members, were either both assigned to the central sample, if $\Delta M_{r}$ between them was less than 0.5 , or the dimmer of the pair was assigned to the satellite sample otherwise. Practically identical results were obtained with both separation criteria. The results presented here use the first.

The absolute magnitudes in the NYU-VAGC sample used in Berlind et al. (2006) are $k$-corrected and corrected for passive evolution to $z=0.1$ (Blanton et al. 2003c, 2003d). These corrected magnitudes are used here. Membership in the red sequence is defined using the $M_{r}$-dependent color cut presented in Li et al. (2006) for the SDSS; $g-r>-0.788-0.078 M_{r}$. The $i$-band Sérsic index, $n_{i}$, is defined as $I_{i}(r)=A \exp \left[-\left(r / r_{0}\right)^{\left(1 / n_{i}\right)}\right]$ and its measurement is discussed in Blanton et al. (2003b). Galaxies with de Vaucouleurs profiles are assigned $n_{i}=4$, galaxies with exponential disk profiles are assigned $n_{i}=1$, and galaxies with late- and early-type light profiles can be separated using $n_{i} \approx 2.5$. As discussed in Blanton et al. (2003b), the Sérsic index is not biased by seeing, which is an improvement over other measures of the light profile such as the concentration. Both seeing and the non-axisymmetric shapes of real galaxies introduce scatter into the measurement of $n_{i}$.

While estimated stellar masses are available for most of the galaxies in the group catalog, results are presented in terms of $M_{r}$. That is, galaxies are separated according to the mass of the group in which they reside, $M_{\mathrm{gr}}$, and their luminosity, as measured by $M_{r}$. The volume-limited sample used to identify the groups was luminosity-selected. Using $M_{r}$ therefore avoids completion issues. Within a volume-limited, luminosityselected sample the $M_{r}$ cut at low luminosity translates into a color cut that varies with stellar mass. Using stellar mass would therefore introduce strong, unacceptable biases into the work presented here. The choice to sidestep these issues by using luminosity is justified by recent work that compares $N$-body simulations to galaxies in the SDSS and that indicates that luminosity is a fair measurement of the maximum circular velocity of the dark matter halos (Tasitsiomi et al. 2004). The maximum circular velocity is directly related to the depth of the gravitational well in the halo and therefore to the binding force between the body of the galaxy and the gas disk and galactic halo.

Finally, I chose to use the fraction of blue galaxies that transition to the red sequence to probe the SFH of the satellite galaxies. This has clear advantages. The split between the red sequence and the blue cloud is a relatively clean indicator of the presence, or absence, of recent star formation. Within the red sequence and the blue cloud the average colors of galaxies are 

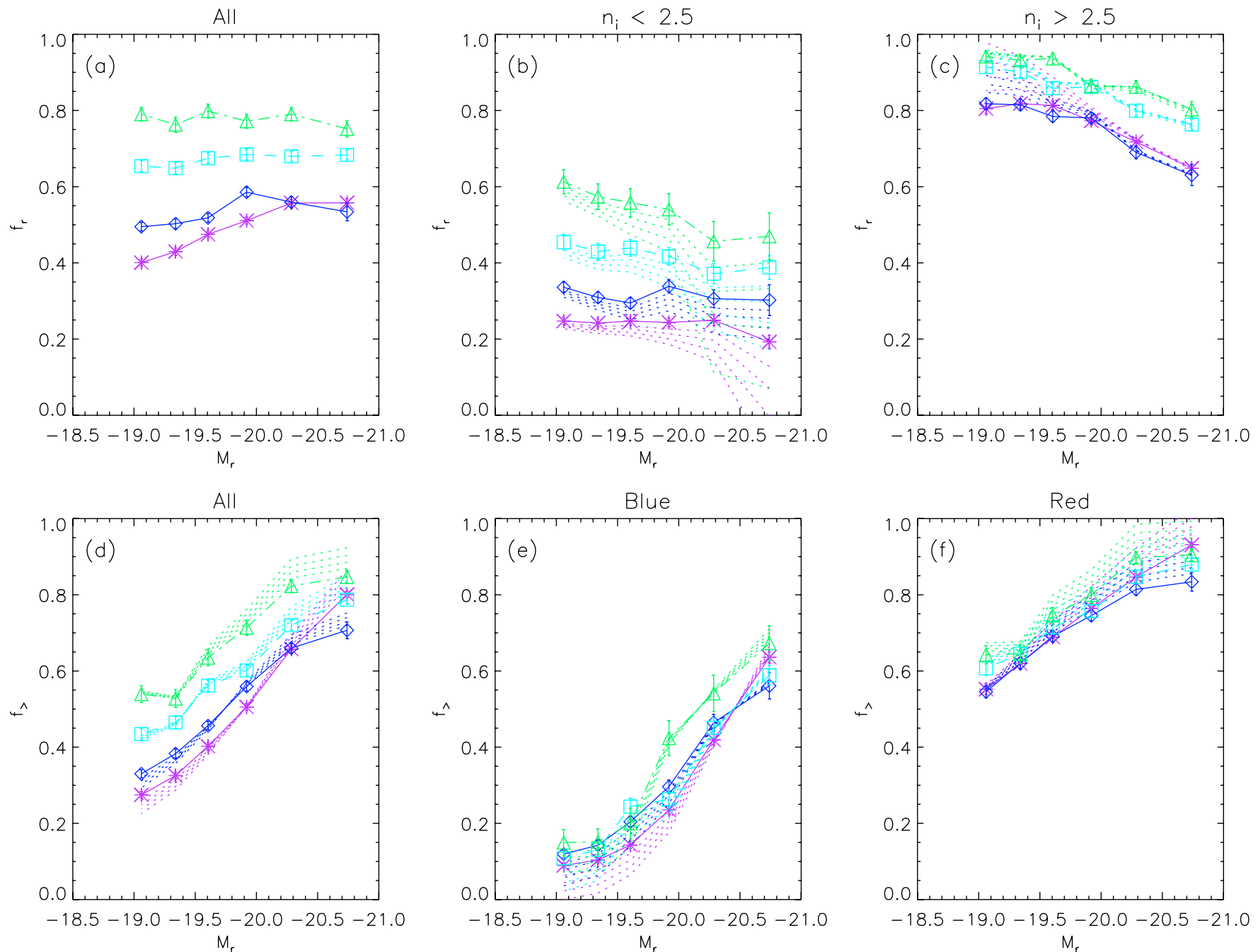

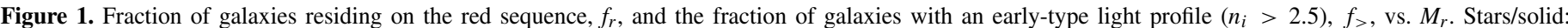

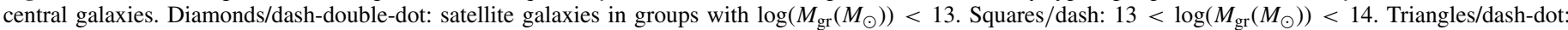

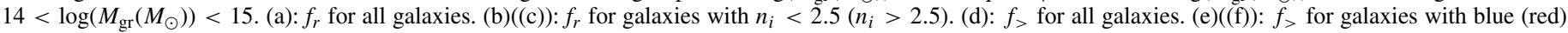

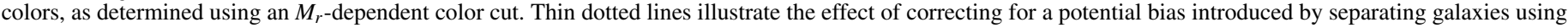

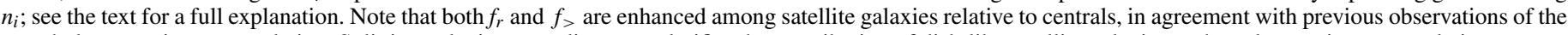

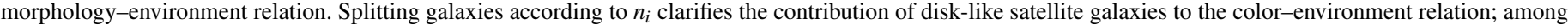
galaxies with disk-like light profiles $\left(n_{i}<2.5\right), f_{r}$ increases with $M_{\mathrm{gr}}$.

(A color version of this figure is available in the online journal.)

only weakly correlated with either host group mass or local environment (Balogh et al. 2004; Weinmann et al. 2006a). Hence, all of the available relevant information is captured in the fraction of galaxies which reside on the red sequence, hereafter the red fraction. Focusing on the red fraction also simplifies comparisons with previous related studies that make use of the bimodality in galaxy colors.

\section{RESULTS AND ANALYSIS}

This section presents an analysis of the red fraction and the fraction of galaxies with early-type light profiles as a function of galaxy luminosity, group membership, and group mass. In Figure 1(a), the red fraction, $f_{r}$, is plotted versus the $r$-band absolute magnitude, $M_{r}$. The four lines correspond to all central galaxies and to satellite galaxies in groups of $\log \left(M_{\mathrm{gr}}\left(M_{\odot}\right)\right)<13,13-14$, and 14-15. In Figure $1(\mathrm{~d})$, the fraction of galaxies with an $i$-band Sérsic index, $n_{i}$, greater than 2.5, $f_{>}$, is plotted in the same manner. Figures 1(b) and 1(c) show $f_{r}$ versus $M_{r}$, but only include galaxies with $n_{i}<2.5$ and $n_{i}>2.5$, respectively. Figures $1(\mathrm{e})$ and $1(\mathrm{f})$ plot $f_{>}$versus $M_{r}$ for galaxies with blue (red) colors, as determined using the $M_{r}$-dependent color cut. Galaxies are binned in $M_{r}$ such that in the intermediate $M_{\mathrm{gr}}$ bin each $M_{r}$ bin has an equal number of blue galaxies, resulting in uniform measurement errors across the luminosity range. The errors shown in Figure 1 are given by $\sigma^{2}=f(1-f) / N$. These errors were propagated to determine the errors for Figures 2 and 3.

\subsection{Comparison to Previous Results}

The results presented in Figure 1 are in agreement with previous observations. Both $f_{r}$ and $f_{>}$are higher among satellite galaxies than among central galaxies with the same luminosity, Figures 1(a) and 1(d), coinciding with previous observations of the morphology-environment relation. This enhancement is reflected in the correlation of both color and structure with local environment, as well as in the relatively strong clustering of both red and concentrated galaxies. In addition, $f_{r}$ increases with $M_{\mathrm{gr}}$, 
All
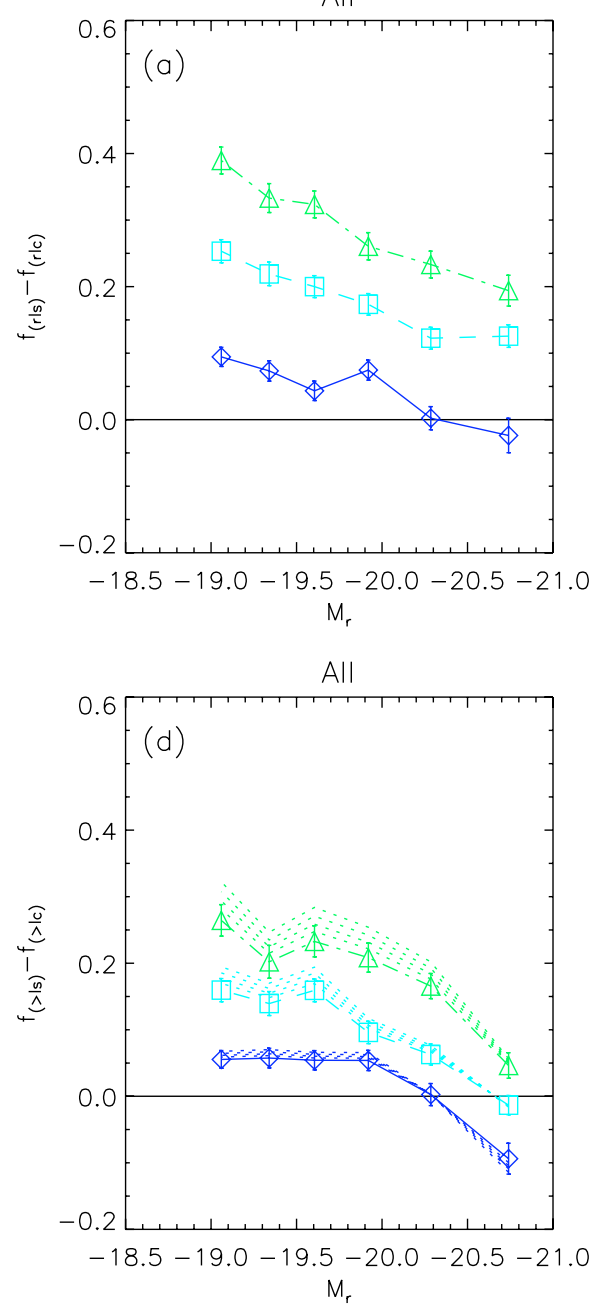
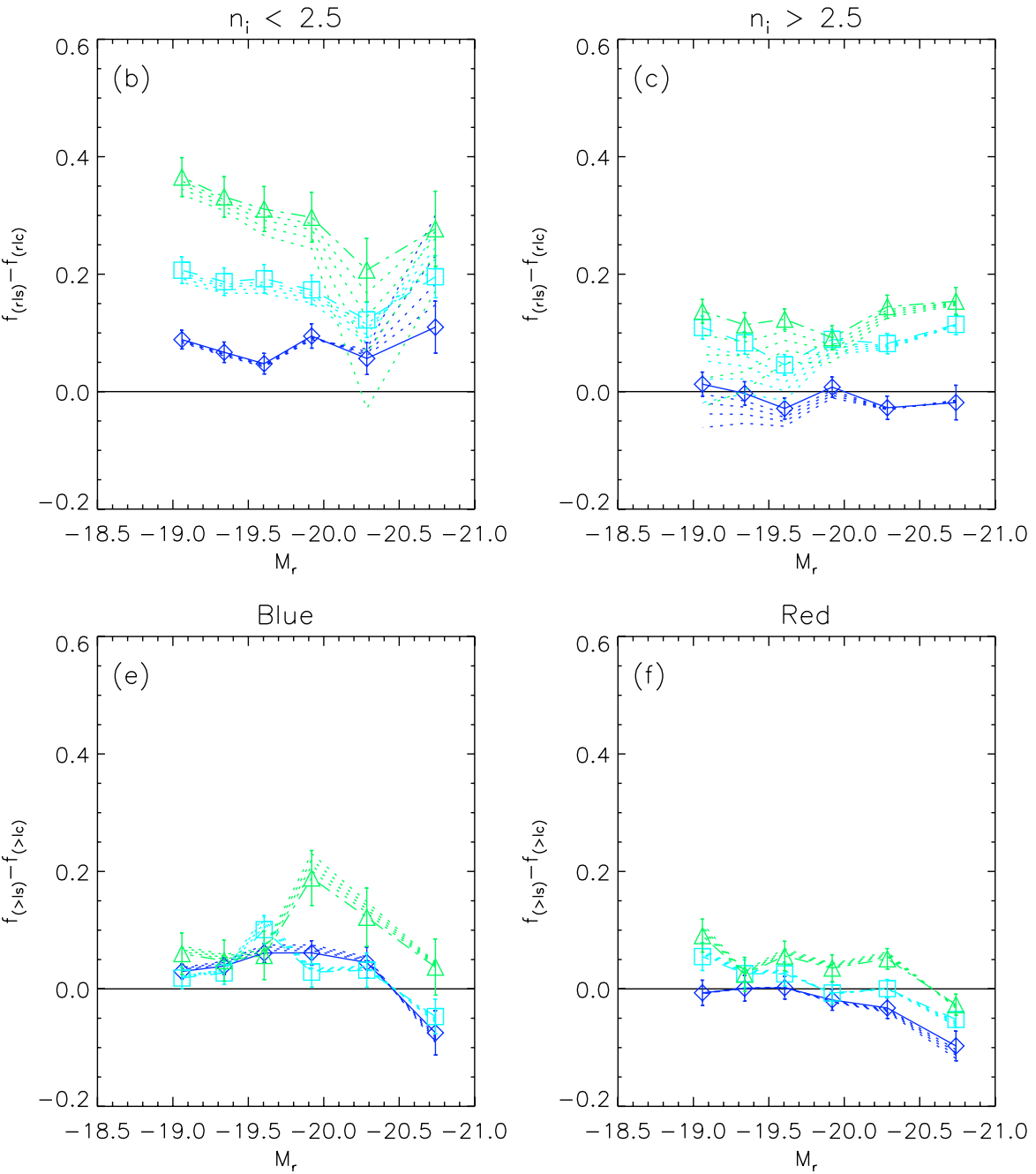

Figure 2. Difference between satellite galaxies and central galaxies in the red fraction, $f_{(r \mid s)}-f_{(r \mid c)}$, and in the fraction of galaxies with $n_{i}>2.5, f_{(>\mid s)}-f_{(>\mid c)}$. Symbols, line styles, and panels are the same as in Figure 1. Note that an excess of red galaxies is observed among the satellite galaxies in both of the $n_{i}$-selected sub-populations, but a similar excess is not seen for $f_{>}$in either the blue or red sub-populations. This asymmetry, which has been observed before, suggests that an interaction between satellite galaxies and the IGM is suppressing star formation among disk-like satellite galaxies.

(A color version of this figure is available in the online journal.)

as observed previously. Note that $f_{>}$also increases with $M_{\mathrm{gr}}$. Among central galaxies $f_{r}$ increases with luminosity, as has often been observed. Among all satellite galaxies, however, $f_{r}$ appears to be independent of $M_{r}$, which was noted previously by Weinmann et al. (2006a).

The previously observed asymmetry between color and structure is apparent in Figure 1. The increase in $f_{r}$ among satellite galaxies is larger than the similar increase in $f_{>}$. The true strength of the asymmetry is seen clearly when Figures 1(b) and 1(e) are compared. Among disk-like galaxies, $f_{r}$ is significantly enhanced for satellite galaxies, Figure 1(b). In contrast, among blue galaxies, there is no significant difference in $f_{>}$between central and satellite galaxies, Figure 1(e). Additionally, among elliptical galaxies $\left(n_{i}>2.5\right), f_{r}$ is higher for satellite galaxies while among red galaxies $f_{>}$is independent of group environment, Figures 1(c) and 1(f). This is the perfect asymmetry between color and structure observed by Quintero et al. (2006).

\subsection{Disk-like Satellites}

In order to constrain models of star formation fueling in satellite galaxies, this section focuses on the fraction of galaxies that transition from the blue cloud to the red sequence as a function of group mass and satellite luminosity for galaxies with late-type and early-type light profiles separately. These results primarily test for physical processes that affect SFRs, and thereby colors, without altering structure, for example, stripping of the galactic halo and the gas disk. The primary focus is on determining $f_{r}$ among satellites with late-type light profiles.

Measuring $f_{r}$ as a function of $M_{\mathrm{gr}}$ and satellite luminosity, $L_{\text {sat }}$, is a useful probe of the physics under consideration. The efficiency of both disk stripping and galactic halo stripping depends on $M_{\mathrm{gr}}$, which sets the strength of the stripping wind, and the depth of the galactic potential, of which $L_{\mathrm{sat}}$ is a measure and which determines the binding force between the galaxy and its gas. Under conditions where stripping may occur, satellites can be split into three regimes. At the smallest $L_{\mathrm{sat}} / M_{\mathrm{gr}}$, stripping is extremely effective, and all satellites are completely stripped. At the largest $L_{\text {sat }} / M_{\text {gr }}$ stripping is equally ineffective. At intermediate $L_{\mathrm{sat}} / M_{\mathrm{gr}}$, partial stripping occurs with an efficiency that correlates with both $M_{\mathrm{gr}}$ and $L_{\mathrm{sat}}$. A correlation between $f_{r}$ among satellite galaxies and both $M_{\mathrm{gr}}$ and $L_{\text {sat }}$ is an observational signature of partial stripping, an expectation that was confirmed with SAMs (e.g., Font et al. 2008). 


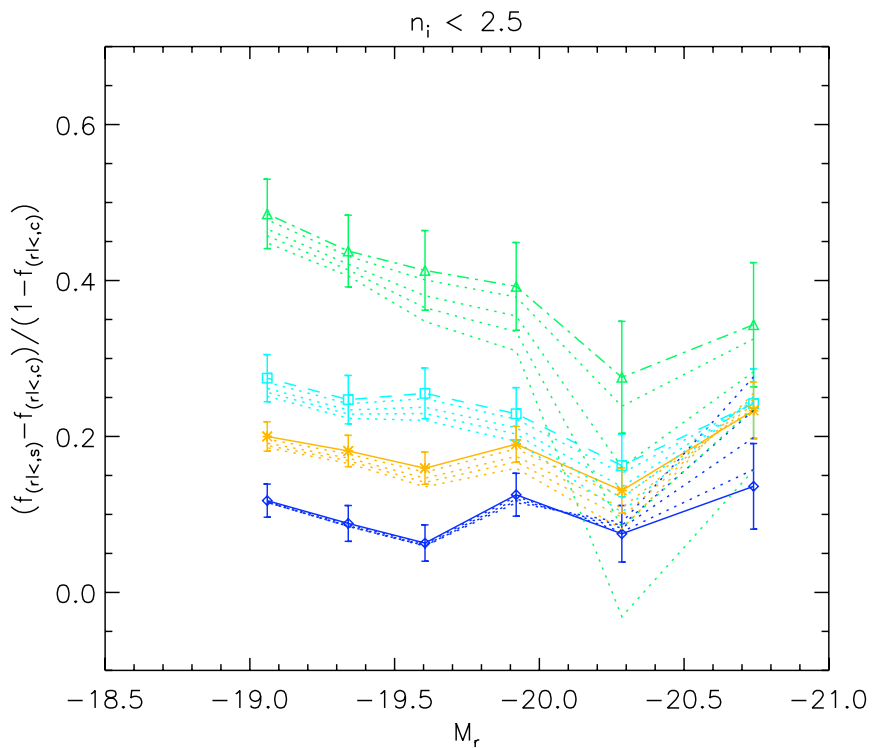

Figure 3. Normalized difference in $f_{(r \mid<)}$ between satellite and central galaxies, $\left(f_{(r \mid<, s)}-f_{(r \mid<, c)}\right) /\left(1-f_{(r \mid<, c)}\right)$. This is an estimate of the fraction of satellites accreted as blue star-forming galaxies that have since transitioned to the red sequence. Most symbols and line styles are the same as in Figure 1. Stars/solid: normalized difference without separating satellites by $M_{\mathrm{gr}}$. Note that in this case no dependence on $M_{r}$ is seen, consistent with the results of van den Bosch et al. (2008).

(A color version of this figure is available in the online journal.)

The observations presented here are consistent with partial stripping occurring among disk-like satellite galaxies. The enhancement in $f_{r}$ among satellites with late-type light profiles increases with $M_{\mathrm{gr}}$. Figure 2 shows the difference in $f_{r}$ and $f_{>}$ between the satellite galaxies and central galaxies of the same luminosity, $f_{s}-f_{c}$, here on referred to as $\Delta f_{r}$ and $\Delta f_{>}$. The full satellite population clearly shows a decline in $\Delta f_{r}$ with luminosity for all $M_{\mathrm{gr}}$. Galaxies with late-type light profiles show a decline in $\Delta f_{r}$ with luminosity in the most massive groups, may show a slight decline in the middle $M_{\mathrm{gr}}$ bin, and show no dependence on luminosity for the lowest mass groups. As will be shown in the next section (Section 4.3), the weakness of this trend is consistent with partial RPS of the extended $\mathrm{HI}$ disk.

Disk-like satellite galaxies make a significant contribution to the discrepancy between conventional SAMs and observations. They show a stronger correlation between $f_{r}$ and $M_{\mathrm{gr}}$ than galaxies with $n_{i}>2.5$, make up a significant fraction of the satellite population, and therefore clearly contribute to the increase in $f_{r}$ with increasing $M_{\mathrm{gr}}$ among the full satellite population. This is evidence for a new physical mechanism, such as disk inflow plus partial RPS.

There are two other interesting trends present in Figures 1 and 2 which place constraints on theories of galaxy evolution in groups and clusters. In particular, they constrain the evolution of the colors of elliptical galaxies and of stellar structure. Among galaxies with early-type light profiles, $f_{r}$ is high, as expected, and is enhanced slightly in groups with masses above $10^{13} M_{\odot}$. No other dependence on $M_{\mathrm{gr}}$ is seen. This may indicate that fresh accretion plays a larger role in creating star-forming elliptical galaxies than cooling of the galactic halo. Second, $\Delta f_{>}$increases with $M_{\mathrm{gr}}$ and decreases with luminosity, particularly at the bright end. The behavior of $\Delta f_{>}$and $\Delta f_{r}$ at the bright end may be particularly affected by the separation between satellites and centrals, however, and so should be viewed with skepticism. A slight enhancement of $f_{>}$among the satellites is seen in the blue and red subsamples independently, again primarily at the bright end. It is clear, however, that the majority of the elliptical satellites that are responsible for the correlation between $f_{>}$ and $M_{\mathrm{gr}}$ are red. Whatever mechanism transforms disk galaxies into elliptical galaxies either operates preferentially in groups or operates preferentially on galaxies that are destined to be accreted by groups. Group membership may also, of course, prevent elliptical galaxies from evolving back into disk galaxies.

Separating galaxies according to their light profiles using $n_{i}$ is potentially a powerful way of both distinguishing between processes that do and do not alter structure as well as detecting those that operate exclusively in either disks or elliptical galaxies. One must be careful when attempting this however. The errors on the $g-r$ colors in the SDSS spectroscopic survey are small and unlikely to affect the separation of galaxies into the red sequence and blue cloud. In contrast, scatter in the measured $n_{i}$, due to both seeing and the non-axisymmetric shapes of real galaxies, may lead to biased results when $n_{i}$ is used to separate galaxies based on their stellar structures. Observed trends not only in $f_{>}$itself, but also in $f_{r}$ within $n_{i}$-selected sub-samples can be biased. In the rest of this section, the potential effect on the observations presented here are considered. I find that the measured trends are robust to this effect.

The noise inherent in measuring $n_{i}$ has multiple effects. First, the measured $f_{>}$will tend to be less extreme than the true $f_{>}$. As $f_{>}$increases, the fraction of galaxies with true $n_{i}>2.5$ that scatter out of the sample with measured $n_{i}>2.5$ increases relative to the fraction of galaxies that scatter into it, and vice versa. Furthermore, as the red fraction in the $n_{i}>2.5$ population is significantly higher than in the $n_{i}<2.5$ population, scattering of galaxies between these two sub-populations will cause $f_{r}$ to be overestimated for galaxies with $n_{i}<2.5$ and underestimated for galaxies with $n_{i}>2.5$. More to the point, the size of this effect varies with $f_{>}$and consequently correlations between $f_{>}$ and $M_{\mathrm{gr}}$ or luminosity can induce correlations in the measured $f_{r}$ among galaxies selected on $n_{i}$.

It is difficult to characterize for an individual galaxy the "error" in $n_{i}$. There are long tails in the likelihood distribution and significant correlations with the light profile scale length, $r_{0}$ (see Blanton et al. 2003b). Therefore, in order to estimate the impact of the above effects on the results presented here the following simplified treatment is adopted. Let $n_{i t}$ and $n_{i m}$ be the true and measured $n_{i}$ of a galaxy, respectively. Let $n$ be the percentage of galaxies with $n_{i t}>2.5$ that scatter into the $n_{\text {im }}<2.5$ population:

$$
n \equiv \frac{N\left(n_{i m}<2.5 \mid n_{i t}>2.5\right)}{N\left(n_{i t}>2.5\right)} .
$$

Let $t$ be the percentage of galaxies with $n_{i t}<2.5$ that scatter in the opposite direction. Let $f_{>t}$ be the fraction of galaxies with $n_{i t}>2.5$ and $f_{>m}$ the fraction with $n_{i m}>2.5$. Then $f_{>m}$ and $f_{>t}$ are related by

$$
f_{>m}=(1-n) f_{>t}+t\left(1-f_{>t}\right)
$$

or

$$
f_{>t}=\frac{f_{>m}-t}{1-(n+t)} .
$$

Let $f_{(r \mid<(>)) t}$ be the true $f_{r}$ in the $n_{i}<(>) 2.5$ population and $f_{(r \mid<(>)) m}$ be the measured $f_{r}$. With these definitions

$$
f_{(r \mid<) m}=\frac{N_{(r,<) m}}{N_{<m}}=\frac{f_{(r \mid<) t}\left(1-f_{>t}\right)(1-t)+f_{(r \mid>) t} f_{>t} n}{\left(1-f_{>t}\right)(1-t)+f_{>t} n}
$$


or

$$
f_{(r \mid<) t}=f_{(r \mid<) m}-\frac{n}{(1-t)} \frac{f_{>t}}{\left(1-f_{>t}\right)}\left(f_{(r \mid>) t}-f_{(r \mid<) m}\right) .
$$

Introducing the corresponding equations for $f_{(r \mid>) t}$ followed by some algebra gives

$$
\begin{aligned}
f_{(r \mid<) t} & =\left(f_{(r \mid<) m}-\frac{n}{(1-t)} \frac{f_{>t}}{\left(1-f_{>t}\right)}\left(f_{(r \mid>) m}-f_{(r \mid<) m}\right)\right. \\
& \left.-\frac{n t}{(1-n)(1-t)} f_{(r \mid<) m}\right)\left(1-\frac{n t}{(1-n)(1-t)}\right)^{-1},
\end{aligned}
$$

where $n t /((1-n)(1-t)) \ll 1$ (see below). By combining Equations (2) and (5), and a symmetric relation for $f_{(r \mid>) t}$ the true values can all be estimated given $n$ and $t$. Given the relatively small errors in color, changes in $f_{r m}$ in the full galaxy sample reflect true differences, galaxies can be cleanly separated by color, and therefore changes in $f_{r}$ do not induce changes in $f_{>m}$.

The fundamental assumption behind this treatment is that at fixed $n_{i}$, and fixed $M_{r}$, the measurement of $n_{i}$ is unaffected by the $g-r$ color. A possible variation with $M_{r}$ is treated separately. There is no reason at this time to assume there is such a correlation; to reiterate, the key is the distribution of measured $n_{i}$ at fixed true $n_{i}$. Galaxies with low $n_{i}$ may show a broader distribution in their measured $n_{i}$ than those with large $n_{i}$, or vice versa. The question is whether galaxies with the same true $n_{i}$ but different $g-r$ have fundamentally different light profiles in the $i$ band. Given the importance of this issue for any investigation of the relationships between $n_{i}$ and color, luminosity, and/or environment; including those presented here and elsewhere in the literature; this point should perhaps be investigated further.

Within this framework, one can constrain $n$ and $t$ by requiring that the various $f$ are all between zero and one, which they must be by definition. Meaningful limits can be placed on both $n$ and $t$ at the faint end of the luminosity range, and even stricter limits are required at the bright end. At fixed luminosity, $n$ and $t$ are presumably independent of $M_{\mathrm{gr}}$, but one might expect that $n$ and $t$ decrease with luminosity. At the faint end, $t$ is constrained to be $\leqslant 0.09$ by setting $f_{(>\mid b)}=0$. This constraint is independent of the assumed $n$. Also at the faint end, requiring $f_{(r \mid>) t} \leqslant 1$ and setting $t=0$ places the constraint that $n \leqslant 0.17$. The greatest allowed adjustments across the full range of observations occur when $t=0.09$ and $n=0.1$. These values are inconsistent with the measured $f$ at the bright end, where $f_{(>\mid r)}$ and $f_{(r \mid<)}$ place tighter constraints on $n$ and $t$. Setting $n=t=0.07$ is consistent with the high luminosity $f$.

For the purposes of illustration, I adopt $n=t$ and in Figures 1 and 2 the dotted lines show corrected results for $n=t=(0.03,0.05,0.07,0.09)$. Increasing $n$ increases the size of the correction. In a visual inspection of the galaxies, it appears that $n<t$, that is, a higher fraction of disk galaxies scatter into the $n_{i}>2.5$ population than vice versa. This is misleading, however, as spiral galaxies are relatively easy to identify visually while $\mathrm{S} 0$ and $\mathrm{E}$ are difficult to distinguish. The greatest corrections to the data occur when $n=t$, and in what follows $n=t=0.09$ is adopted as a worse case scenario.

When the galaxies in the sample are grouped by $M_{r}$, a secondary assumption arises that the breadth of the two $n_{i}$ distributions, for galaxies with late- and early-type light profiles, are independent of $M_{r}$. From a measurement perspective, each luminosity bin mixes galaxies with different observed $m_{r}$, with varied shapes, observed under varying conditions. The measurement component of the width is likely insensitive to $M_{r}$. The underlying physical distributions may, however, narrow with luminosity. Less massive galaxies are easier to perturb, which may be reflected in their $n_{i}$ distributions. Because the limit $n=t=0.09$ is set at the faint end, it is a real upper limit across the full luminosity range. Below I briefly consider allowing $n$ and $t$ to decrease with luminosity.

The observations presented at the beginning of this section are robust to corrections for scatter between the two $n_{i}$ populations. As can best be seen in Figure 2, $f_{>}$is likely slightly more sensitive to both $M_{\mathrm{gr}}$ and luminosity than measured. Despite this, after the correction is applied color is still more sensitive to environment than stellar structure and a nearly perfect asymmetry exists between color and structure. In particular, when $n$ and $t$ are set to $0.09, f_{(r \mid<) t}$ increases with $M_{\mathrm{gr}}$, as can be seen in Figures 1 and 2 .

Of special interest is the effect on the observed correlation between $f_{(r \mid<)}$ and luminosity. In general, scatter between the populations induces $\delta f_{(r \mid<)}$ which mimic $f_{>}$, where $\delta f_{(r \mid<)} \equiv$ $f_{(r \mid<) m}-f_{(r \mid<) t}$. Contrary to this expectation, while $f_{>}$increases with $L_{\text {sat }}$ (Figure 1(d)), $f_{(r \mid<) m}$ in the highest $M_{\mathrm{gr}}$ bin decreases with $L_{\text {sat }}$ while $f_{(r \mid<) m}$ in the intermediate and lower $M_{\mathrm{gr}}$ bins is flat (Figure 1(b)). As luminosity increases, however, the scatter between the two populations may decrease, tending to decrease the induced $\delta f_{(r \mid<)}$. The most extreme case allowed by the data would be to assume that $n=t=0$ in the brightest bin, that is, there is no scatter, while in the lowest luminosity bin $n=t=0.09$. With these assumptions, $f_{(r \mid<) t}$ in the highest $M_{\mathrm{gr}}$ bin still decreases with luminosity while $f_{(r \mid<) t}$ does not correlate with $M_{r}$ in the middle and low $M_{\mathrm{gr}}$ bins.

The assumption that $n$ and $t$ vary from their maximum value to 0 as $L_{\text {sat }}$ increases is likely too extreme. Detailed observations reveal star formation in some elliptical galaxies (Salim \& Rich 2010), so $t$ is likely less than $0.09\left(f_{(>\mid b)}>0\right)$. Similarly, it is unlikely that $n$ and $t$ are ever zero. Under the assumption that $n$ and $t$ are constant with luminosity, a small scatter between the two $n_{i}$ sub-samples can disguise a decrease in $f_{(r \mid<) t}$ with luminosity because $f_{>}$increases steeply with luminosity. In this case, the greatest allowed corrections to the data are found for $n=t=0.07$, and $f_{(r \mid<) t}$ decreases with luminosity in all three $M_{\mathrm{gr}}$ bins, as well as for central galaxies. Assuming $n=t=0$, $f_{(r \mid<) t}$ decreases with luminosity in the two higher $M_{\mathrm{gr}}$ bins but not in the lowest $M_{\mathrm{gr}}$ bin. In each case in which a correlation is observed, the measurement errors are marginally inconsistent with no correlation. In at least the highest mass groups $f_{(r \mid<) t}$ decreases with luminosity, as observed in Figure 1. In the middle $M_{\mathrm{gr}}$ bin, a true correlation likely exists which may be partially hidden by scatter in the measured $n_{i}$. There may or may not be a true correlation in the lowest $M_{\mathrm{gr}}$ bin or for the central galaxies, depending on the true values of $n$ and $t$.

Galaxies that are not forming stars when they are accreted by a group or cluster likely remain that way. Differences in $f_{r}$ between satellite and central galaxies are therefore due to satellites that transition from the blue cloud to the red sequence after they are accreted. The most physically motivated measure of the correlation between color and environment is therefore the fraction of blue accreted galaxies that make this transition. This fraction can be estimated by dividing the observed $\Delta f_{(r \mid<)}$ by the blue fraction among the central galaxies, that is, $\left(f_{(r \mid<, s)}-f_{(r \mid<, c)}\right) /\left(1-f_{(r \mid<, c)}\right)$. This normalized difference is only a rough estimate of the fraction of the blue 
accreted galaxies which have transitioned to the red sequence as satellites as it assumes that when the satellite galaxies where accreted they had the same $f_{r}$ as the central galaxies do currently.

This normalized difference among disk-like satellite galaxies, uncorrected and corrected for $n=t=(0.03,0.05,0.07,0.09)$, is shown in Figure 3. It clearly increases with $M_{\mathrm{gr}}$, decreases with luminosity in the highest $M_{\mathrm{gr}}$ bin, likely decreases with luminosity in the mid- $M_{\mathrm{gr}}$ bin, and is flat for the lowest $M_{\mathrm{gr}}$ bin. The observed correlations with luminosity are marginally significant. For the highest $M_{\mathrm{gr}}$ bin, excluding the brightest $M_{r}$ bin there is a $\approx 2.7 \sigma$ decrease in $\Delta f_{r}$ across the $M_{r}$ range and a $\approx 2.3 \sigma$ decrease in the normalized difference. Given the increase in $f_{>}$with luminosity, this is a lower limit on the true luminosity dependence. The relative slopes of the normalized difference versus $M_{r}$ across $M_{\mathrm{gr}}$ are robust to the correction for noise in measuring $n_{i}$. The normalized difference, or transition fraction, was also shown in Hester (2006b) and van den Bosch et al. (2008). van den Bosch et al. (2008) saw no dependence on $M_{r}$, but this may be due to simple differences in methodology (see Figure 3). The observed correlations are generally consistent with partial stripping, as will be discussed in more detail in the following section.

\section{DISCUSSION}

New results using the SDSS have been presented here, which place new constraints on galaxy evolution. They explore the correlation between morphology and environment while distinguishing both between processes that do and do not affect structure and between processes that occur in disk galaxies and in elliptical galaxies. This study was motivated by the inability of conventional SAMs (e.g., Bower et al. 2006; Croton et al. 2006; Weinmann et al. 2006b) that rely on complete galactic halo stripping to account for the fraction of star-forming satellites in groups in a way that is consistent with observed galaxies or with the latest generation of cosmological simulations of gas accretion onto galaxies. By clarifying the contribution of disk galaxies to $f_{r}$, these results challenge the hypothesis that partial stripping of the galactic halo accounts for the discrepancies between conventional SAMs and observations. In its place, I advance the hypothesis that inflow occurs within the neutral atomic gas disk that extends beyond the stellar disk of most disk galaxies and that partial RPS of this disk causes the observed correlations between $f_{r}$ and group mass among disk galaxies. This section discusses this mechanism in more detail and places it in the context of the observed groups.

\subsection{RPS and the SFR}

Gunn \& Gott (1972) first proposed RPS to explain an observed deficiency of gas-rich galaxies in clusters. By comparing the RP generated as a satellite galaxy orbits supersonically through the intracluster medium (ICM) to the gravitational attraction between the stellar and gas disks, they concluded that RPS can remove the gas disk from spiral galaxies as they pass through the center of a cluster. Subsequent observations support this theory. Galaxies in nearby clusters are observed to be deficient in $\mathrm{HI}$ and to have truncated gas disks when compared to field galaxies of similar morphology and optical size (Bravo-Alfaro et al. 2000; Cayatte et al. 1994; Giovanelli \& Haynes 1983; Solanes et al. 2001). In addition, asymmetric extraplanar gas that appears to have been pushed out of the disk is observed in several Virgo spirals (Kenney \& Koopman 1998; Kenney et al. 2004a, 2004b; Chung et al. 2007). An undisturbed stellar disk accompanied by a truncated gas disk and extraplanar gas is a strong indication that the galaxy's gas disk is interacting with the ICM. RPS has also been repeatedly observed in simulations of disk galaxies in an ICM wind (Abadi et al. 1999; Quilis et al. 2000; Schulz \& Struck 2001; Marcolini et al. 2003; Roediger \& Hensler 2005; Roediger \& Brüggen 2007).

Modeling predicts that MW-like galaxies in groups retain the gas within the stellar disk while the regions of the $\mathrm{H}$ I disk that extends beyond the stellar disk are partially RP-stripped. Hester (2006a) presents a detailed analytical model of RPS based on comparing the RP to the gravitational attraction between the gas disk and its galaxy. It reproduces both observations and simulations across a range of RP regimes, including simulations of RPS from low RPs (Roediger \& Hensler 2005). It predicts partial stripping of MW-like galaxies in groups. The complete stripping that occurs under high RPs truncates star formation. It is unknown, however, how partial RPS of the extended H I disk may affect star formation.

Two gigayears is a typical gas consumption time within the optical disks of normal spiral galaxies, though gas recycling may increase this time by a factor of 2-3 (Kennicutt 1998). A spiral galaxy is unlikely to consume the entirety of the gas within its optical disk, however. When the gas surface density drops below a critical value, the star formation efficiency declines dramatically. This effect is seen on the edges of the optical disks of normal spiral galaxies, where the star formation law shows a sharp transition, and in low surface brightness galaxies (see, e.g., Martin \& Kennicutt 2001; Thilker et al. 2007; Bigiel et al. 2008; Wyder et al. 2009). The gas densities within the optical disk are typically close to this critical density (see Figure 8 of Martin \& Kennicutt 2001). Normal spiral galaxies rely on the delivery of new gas into the optical disk in order to continue forming stars at their current rate. A central galaxy can sustain star formation through the infall of new gas and the continual cooling of the hot galactic halo. After accretion, infall ceases. In spiral galaxies, the hot galactic halo is likely completely stripped, as discussed above. Nevertheless, comparing observations to conventional SAMs indicates that satellite galaxies have access to a gas reservoir.

Rough estimates indicate that in environments with moderately efficient RPS the gas in the unstripped portion of the extended disk can significantly extend the time for which a satellite can continue to form stars. A typical SFR for an MW-like galaxy is $1 M_{\odot} \mathrm{yr}^{-1}$, corresponding to a gigayear of star formation for every $10^{9} M_{\odot}$ of gas consumed. To estimate the gas mass in a moderately stripped $\mathrm{H}$ I disk, a disk with $\sigma_{H}=20 M_{\odot} \mathrm{pc}^{-2} \exp \left(-R / 4.5 R_{D}\right)$, that is with a scale length and surface density consistent with the galaxies in Swaters et al. (2002), that begins at the optical radius, or $3 R_{D}$. The optical disk scale length for the MW-like model of Hester (2006a) is $R_{D}=5 \mathrm{kpc}$. (In Section 4.3, I retain the assumption of a flat H I disk as was used in Hester 2006a. The model of Hester 2006a correctly predicts the $\mathrm{H}$ I deficiencies of observed galaxies, and this verification is more important to the discussion in Section 4.3 than the particular shape of the $\mathrm{H}$ I profile.) Defining the size of the $\mathrm{H}$ I disk as the radius at which $\sigma_{H}=1 M_{\odot} \mathrm{pc}^{-2}$, this disk extends to $67.4 \mathrm{kpc}$ and contains $4.2 \times 10^{10} M_{\odot}$ of gas. If this gas disk is stripped down to $22.5 \mathrm{kpc}$, or one gas disk scale length, then it retains $7.6 \times 10^{9} M_{\odot}$ of gas. It is unlikely that all of this gas is available to fuel star formation, but a moderate amount of inflow within a truncated disk could extend star formation for several gigayears and reconcile theory with observations. 
Any source of viscosity in a rotating gas disk will facilitate the radial transport of mass and angular momentum within the disk. For a disk with a flat rotation curve, the result will be to transport mass inward and angular momentum outward. This viscosity may be due to thermal or turbulent motions (e.g., McNally et al. 2009), be the result of interactions with magnetic fields or spiral density waves (e.g., Combes 2007), or arise from an unidentified mechanism (see also Shu 1992).

There are theoretical reasons to adopt a model of galaxy formation and evolution that includes inflow within the gas disk. Models that feed star formation with a viscous disk can produce exponential stellar profiles (Lin \& Pringle 1987a, 1987b; Bell 2002; Olivier et al. 1991). These models are relatively insensitive to the initial conditions of the gas disk (Olivier et al. 1991), which is an advantage in the messy cosmological context of hierarchical structure formation with the potential of both hot and cold gas accretion (Kereš et al. 2005; Dekel \& Birnbiom 2006, for example). The extended Hi disk also presents a larger physical cross section to infalling gas and is likely better matched to it in angular momentum (see, for example, Kereš \& Hernquist 2009). Transport within the disk provides accreted gas a means to loose angular momentum and join the star-forming disk.

Fueling star formation with a partially stripped extended $\mathrm{H} \mathrm{I}$ disk has the appropriate characteristics to reconcile conventional SAMs with observations. First, it introduces the required gas reservoir and the mass of this gas supply is significant. In addition, an explicit dependence of the star-forming lifetime of a satellite on group mass is introduced when this disk is partially RP stripped. The stronger the RP or the shallower the gravitational potential of the galaxy, the smaller the stripped, truncated disk and the less fuel available for star formation. Finally, invoking inflow to fuel star formation can be theoretically motivated.

\subsection{RPS and SAMs}

Inflow within the H I disk plus RPS of the extended disk has the right generic behaviors to reconcile conventional SAMs with the observations. This generic consideration does not explicitly consider the effects of tracking satellites' infall histories or their dynamics within their host. The need to increase the time for which satellites form stars appears to be robust to any such considerations. In contrast, the typical time since a satellite was accreted may depend on $M_{\mathrm{gr}}$ and the mass of the satellite, thus alleviating the need for an explicit dependence on $M_{\mathrm{gr}}$, such as is introduced by partial stripping. The efficiency of tidal stripping is proportional to $M_{\mathrm{sat}} / M_{\mathrm{gr}}$, and the typical time since accretion may accordingly increase with $M_{\mathrm{gr}}$. Satellite galaxies are also quickly, compared to dynamical friction, tidally stripped (Boylan-Kolchin et al. 2008) and experience unbound inelastic collisions with each other (Knebe et al. 2006), both of which will tend to decrease any correlation between the typical time since accretion and $M_{\mathrm{gr}}$. The hierarchical growth of structure suggests that satellites in clusters today are more likely to have been in groups several gigayears ago than satellites in groups today are. This could potentially account for the observed correlation with $M_{\mathrm{gr}}$. The best way to address these complicated and intertwined issues is with SAMs.

A SAM that accurately models the galactic halos of spiral galaxies and then introduces RPS of the gas disk is well beyond the scope of this paper. One can, however, compare the inflow plus RPS scenario to the SAMs that have been presented in the literature. The SAMs that include partial galactic halo stripping can reproduce the observed fractions of star-forming satellites as a function of $M_{\mathrm{gr}}$ and satellite luminosity (Font et al. 2008), but do not accurately represent observed or simulated galaxies. Conventional SAMs that rely on complete stripping fail to reproduce the observations. It is likely that a SAM that included disk inflow plus disk RPS would be functionally very similar to those with partial galactic halo stripping, but would be better motivated.

The partial galactic halo stripping scenario is characterized by the following features. In the presence of sufficient fuel, star formation is self regulated, presumably by supernova feedback. After feedback is considered, the net gain in cold gas available for star formation approximately balances the loss to star formation. Following a galaxy's accretion as a satellite, its galactic halo represents a finite gas supply which is consumed by star formation. The rate of cooling in this halo declines as it becomes less massive and therefore less dense. Satellites therefore continue to form stars, but the SFR eventually declines as the galactic halo is consumed.

The disk inflow plus disk RPS scenario shares these features in a functional sense. Models that build exponential disks using inflow must assume a rate of inflow which is similar to the SFR. This is functionally equivalent to self-regulated star formation. After accretion, a stripped disk represents a finite gas supply. The rate of inflow likely depends on the density of the gas disk and will therefore decline over time. This slow starvation, which is a feature of both partial stripping scenarios, is capable of gradually transforming blue spirals into red disks (e.g., Bekki et al. 2002).

There is one final, important, characteristic of the partial galactic halo stripping SAMs. With their current assumptions about the galactic halo density, the SAM satellite galaxies that correspond to the galaxies observed by the SDSS are partially stripped of their galactic halos. Partial stripping is an interesting regime in which galaxies retain gas for star formation and in which the retained gas mass varies with $M_{\mathrm{gr}}$ and satellite luminosity. Furthermore, the efficiency of galactic halo stripping in the SAM is sufficiently sensitive to changes in $M_{\mathrm{gr}}$ and satellite luminosity, that is, increasing $M_{\mathrm{gr}}$ across the observed range of group and cluster masses has a significant impact on the efficiency of stripping and thus on the predicted red fraction. The same holds for satellite luminosity. The next section (Section 4.3) demonstrates that RPS of the extended H I disk shares these characteristics.

\subsection{RPS in the SDSS}

A well-calibrated model of RPS of disk galaxies that can be applied to a wide range of group and satellite masses, which was developed in Hester (2006a), is now used to test whether the observed correlations among disk-like galaxies are consistent with disk inflow plus RPS. The analytical model serves as a basis for a toy model of the red fraction among disk satellites which demonstrates that the observed correlations may reasonably be attributed to disk inflow plus RPS.

Any generic model of RPS will predict that the severity of stripping increases with group mass and decreases with satellite luminosity, and simulations have shown that partial stripping occurs under low RPs (Roediger \& Hensler 2005). The analytical model of Hester (2006a) allows one to improve upon this by estimating the typical efficiency of RPS as function of environment and satellite luminosity as well as the variation about the typical efficiency. Modeling this variation is the key 

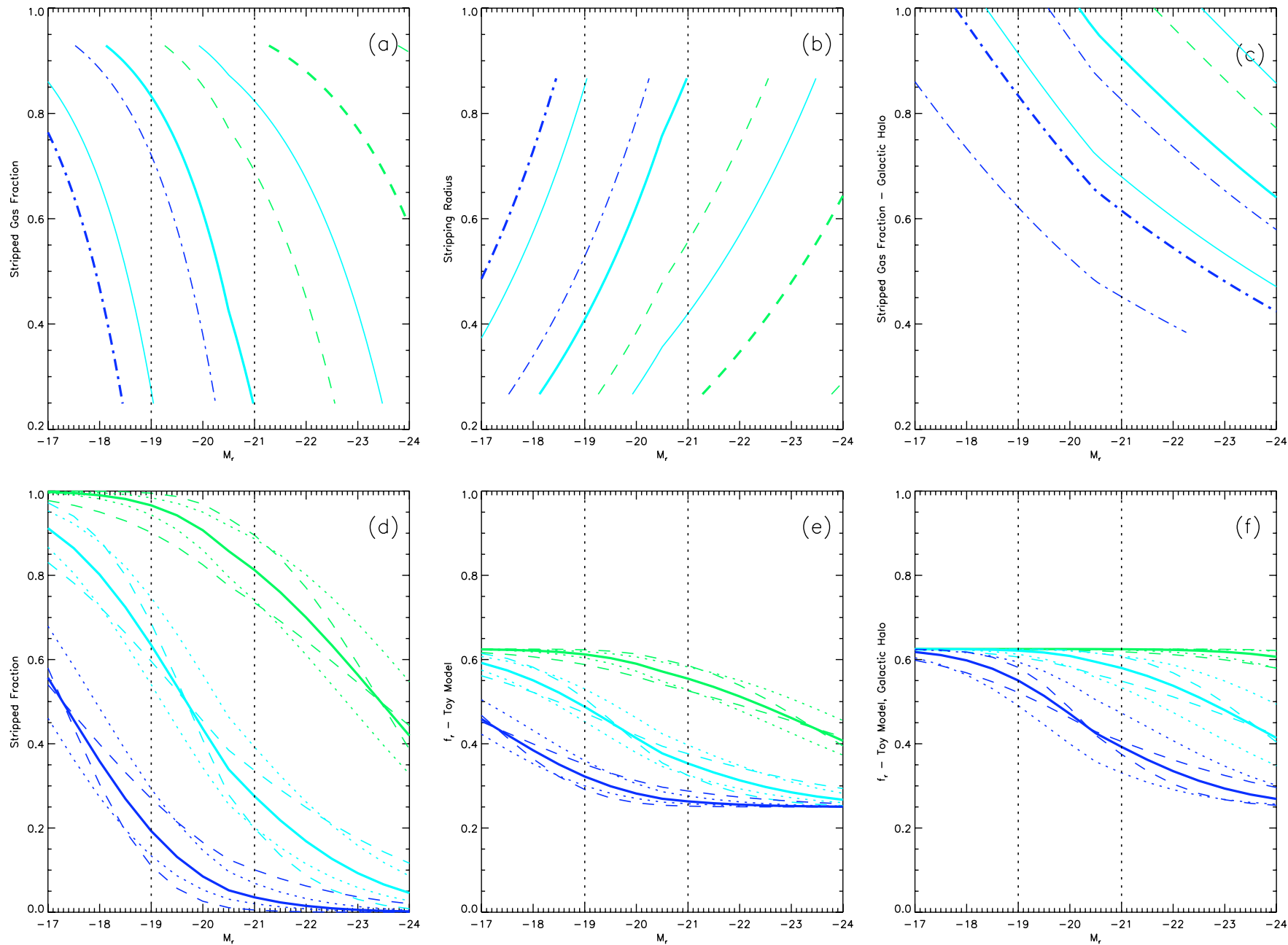

Figure 4. Results from an analytical model of RPS that calculates the fraction of the H I disk that is stripped at pericenter and the radius to which the disk is stripped as a function of $M_{\mathrm{gr}}$ and $M_{r}$. (a): stripped gas fraction. Thick lines correspond to $M_{\mathrm{gr}}=10^{12.25}, 10^{13.25}$, and $10^{14.25} M_{\odot}$ for dash-dotted, solid, and dashed lines, respectively. Thin lines show the variation in the efficiency of stripping. For example, in groups of mass $M_{\mathrm{gr}}=10^{13.25} M_{\odot}$, a satellite galaxy with $M_{r}=-20$ may be stripped as much as one with $M_{r}=-17.5$ or as little as one with $M_{r}=-22.5(\approx 60 \%)$. (b): same as (a) but for the radius to which the disk is stripped scaled by the initial gas disk radius. (c): same as (a) but for the galactic halo. (d): solid lines show the fraction of galaxies that are stripped of at least $70 \%$ of their initial gas disk assuming a Gaussian distribution in $\log \left(M_{\mathrm{eff}}\right)$ with a width of 1 dex for the same $M_{\mathrm{gr}}$ as in (a)-(c) with $M_{\mathrm{gr}}$ increasing upward. See the text for a definition of $M_{\mathrm{eff}}$. Dotted lines demonstrate the effect of varying the stripped gas fraction from $60 \%$ to $80 \%$ and dashed lines show the effect of varying the width between 0.5 and 2 dex. Increasing the stripped gas fraction decreases the fraction of satellites that are stripped while increasing the width decreases the slope of the stripped fraction vs. $M_{r}$. (e): a toy model of $f_{r}$ based on (d) that is matched by eye to the observations by fixing $f_{r}$ for central galaxies to $25 \%$ and the fraction of stripped star-forming galaxies that have transitioned to red sequence as a result of stripping to $50 \%$ of the full stripped fraction. (f): the same toy model but for the galactic halo.

(A color version of this figure is available in the online journal.)

to estimating the sensitivity of the impact of disk RPS to $M_{\mathrm{gr}}$ and $M_{r}$

As will be shown, the predicted underlying dependence of the stripped gas fraction on both $M_{\mathrm{gr}}$ and $M_{r}$ is strong within the SDSS groups, but a large variation in the efficiency of stripping degrades this correlation significantly. A toy model predicting the $f_{r}$ in groups is used to show that the strong observed dependence of $f_{r}$ on $M_{\mathrm{gr}}$ is consistent with an RPS origin despite this scatter. The toy model also reconciles the strong predicted dependence of the typical stripped gas fraction on $M_{r}$ with the weak observed correlation between $f_{r}$ and $M_{r}$, showing that a weak trend is in fact expected.

Figures 4(a) and 4(b) show the analytical model predictions for the stripped gas mass fraction and the radius to which the disk is stripped, scaled by the initial disk radius, as a function of $M_{r}$ for three different group masses. The group masses are $M_{\mathrm{gr}}=10^{12.25}, 10^{13.25}, 10^{14.25}$, typifying the three observed $M_{\mathrm{gr}}$ bins. To convert between stellar mass, which is itself a model parameter, and luminosity, luminosity-dependent mass-to-light ratios in the $r$ band are taken from Figure 14 of Kauffmann et al. (2003a). The scatter in this relation $(\approx 0.15 \mathrm{dex})$ is neglected. To match dark matter halo masses to stellar disk masses, a value of $M_{\mathrm{DM}} / M_{*}=20$ is adopted based on the work of Baldry et al. (2008).

The model of Hester (2006a) focuses on the H I disk that extends beyond the stellar disk. The model is based on calculating the disk radius at which the RP from the ICM wind equals the gravitational restoring "pressure" between the gas disk and the rest of the galaxy. The gravitational restoring pressure is found by placing a flat $\mathrm{H}$ I disk in a gravitational potential consisting of a dark matter halo, a stellar disk, and a stellar bulge. The RP is determined by letting the satellite orbit in a Navarro-Frenk-White (NFW) potential through a $\beta$ profile ICM. The gas fraction that a galaxy is striped of is found to depend on the ratio of the satellite mass to the group mass, $M_{\mathrm{sat}} / M_{\mathrm{gr}}$, and the values of several descriptive model parameters. 
The exact model parameters used for Figure 4 are given in Hester (2006a). The galaxy parameters used are those for a large spiral, with a dark matter halo mass, stellar mass, disk size, and $\mathrm{H}$ I surface density comparable to an MW-like galaxy. The IGM parameters for the lowest mass galaxy groups, $M_{\mathrm{gr}}=10^{12.25} M_{\odot}$, match the observed properties of poor groups with observed X-ray emission (Helsdon \& Ponman 2000; Sanderson \& Ponman 2003; Osmond \& Ponman 2004). The ICM parameters for the higher mass groups correspond to rich groups and poor clusters (Mohr et al. 1999). In Hester (2006a), these are referred to as the "low-mass group model" and the "middle-mass cluster model," respectively. The results shown in Figure 4 assume that the pericenter of the satellite's orbit is 0.35 times the virial radius, which is a typical value in $N$-body simulations (Gill et al. 2004).

The analytical model also includes a galactic halo, the density of which is consistent with the constraints placed by X-ray observations. The galactic halo is modeled by placing gas in hydrostatic equilibrium in the satellite's NFW halo at the halo's virial temperature. The model requires the choice of an inner radius for the galactic halo, which is set to the radius at which the cooling time $(T / \dot{T})$ is $30 \mathrm{kyr}$. This choice essentially defines what is meant by the galactic halo, and hence what is meant by the "fraction" of the halo that is stripped. The absolute gas mass stripped from the galactic halo is not affected as the density in the outer halo, the parameter of relevance for stripping, is fixed by the X-ray observations. The "fraction" of the gas stripped is affected. Selecting a longer cooling time corresponds to a smaller galactic halo, larger stripping fraction, and more "effective" galactic halo stripping. The cooling time reaches $30 \mathrm{kyr}$ at approximately three disk scale lengths. Within this radius and on such short timescales gas likely not well characterized as a hydrostatic galactic halo, but instead likely interacts closely with the disk both hydrodynamically and through star formation and feedback. The RP on the galactic halo is calculated as above, and it is assumed that the galactic halo is stripped down to the radius at which the RP equals the thermal pressure. It is this model that predicts that the galactic halo is efficiently stripped even in environments with low RPs.

For reference, Figure 4(c) shows the mass fraction stripped from the galactic halo for the same $M_{\mathrm{gr}}$ as in Figures 4(a) and 4(b). Galactic halo stripping is significantly more efficient than disk stripping. Note that the model gives lower limits on the stripped fraction as the X-ray observations place upper limits on the density of the galactic halo.

Satellite galaxies of the same luminosity in groups of the same $M_{\mathrm{gr}}$ can nevertheless be stripped of significantly different fractions of their gas disk due to differences in both morphology and orbit. This scatter can be well defined in terms of a satellite's effective mass $M_{\text {eff }}$, where $M_{\text {eff }}$ is the mass of a typical satellite galaxy that would be stripped of the same gas fraction as the satellite in question when on a typical orbit in a typical group with the same $M_{\mathrm{gr}}$ as the satellite. The analytical model parameters are all descriptive parameters which correspond directly to observables such as surface brightness, gas disk density, IGM profile, etc. The scatter between $M_{\text {sat }}$ and $M_{\text {eff }}$ was estimated in Hester (2006a) by introducing variations in these parameters similar to those observed for real galaxies and groups, and an extensive treatment of this estimation can be found there.

The width of the $M_{\text {eff }}$ distribution is roughly an order of magnitude. A $10^{11} M_{\odot}$ galaxy in a group with $M_{\mathrm{gr}}=10^{14} M_{\odot}$ can be stripped as much as a $10^{10} M_{\odot}$ galaxy or as little as a
$10^{12} M_{\odot}$ galaxy in a group of the same mass. This scatter is mainly due to variations in the galaxies' orbits, stellar and $\mathrm{H}_{\mathrm{I}}$ disk scale lengths, and in the density and extent of the groups' IGM and can be split equally between these elements. Galaxies on radial orbits, orbiting face-on, with low surface brightnesses or low gas fractions are more susceptible to RPS than those on circular orbits, orbiting edge-on, with dense stellar and gas disks. Similarly, variations in the density and concentration of the IGM across groups increase the scatter. Galaxies on typical orbits experience less striping if the IGM is less dense or more concentrated.

The thin lines in the top panels of Figure 4 illustrate the effects of the width of the $M_{\text {eff }}$ distribution by showing the $\sim 1 \sigma$ ( $\sim 1$ dex) variation in the $M_{r}$ at which a galaxy is stripped of a given gas fraction or to a given radius. One can begin to see the importance of considering this scatter. Neglecting it, one would conclude that in the least massive groups no stripping is occurring, that all of the satellites in the high-mass groups are completely stripped of at least their extended gas disks, and that partial stripping only occurs in the intermediate mass groups. Once the scatter is considered, however, one sees that some of the satellites in the low-mass groups, those that are particularly susceptible to RPS, are partially stripped, while some of the satellites in the high-mass groups, those that are particularly resistant to RPS, are only partially stripped. Partial stripping is therefore occurring in all three group samples. Naively, from the strong dependence of the typical efficiency of stripping on $M_{r}$ one might predict a similarly strong correlation between $M_{r}$ and $f_{r}$. Once the distribution of $M_{\text {eff }}$ is considered, however, this is no longer the case. This will now be shown with a toy model of $f_{r}$ as a function of $M_{\mathrm{gr}}$ and $M_{r}$.

To construct a very simplified model I assume that all satellite galaxies that have been stripped of at least $x \%$ of the mass in the gas disk have consequently transitioned to the red sequence. All other satellites remain blue. At each $M_{r}$, a Gaussian distribution in $\log \left(M_{\text {eff }}\right)$ with a width of 1 dex is assumed and the fraction of galaxies stripped of greater than $x \%$ of the gas mass in the extended disk is calculated. Comparing Figures 4(a) and 4(b) with the observed $f_{r}$ suggests one should choose a high stripped gas fraction. The observed low-mass groups show little sign of stripping while the high-mass groups show an indicative correlation with $M_{r}$. Accordingly, $x$ is set to $70 \%$. The results of this calculation are shown in Figure 4(d). The same calculation was also done using $60 \%$ and $80 \%$ and using widths of 0.5 and 2 dex. These results are also plotted. A clear dependence of the "stripped fraction" on $M_{\mathrm{gr}}$ is seen and the telltale correlation with $M_{r}$ is also observed for all three $M_{\mathrm{gr}}$. The correlation between the stripped fraction and $M_{r}$ is strongest in the intermediate $M_{\mathrm{gr}}$ bin, but it is not as strong as might have been expected. The strength of the correlation with $M_{r}$ is inversely correlated with the assumed width of the distribution of $M_{\text {eff }}$.

The toy model is taken one step further. First, $25 \%$ of the observed central galaxies with late-type profiles are red. The model is therefore applied to calculate the "red" fraction for $75 \%$ of the galaxies while the other $25 \%$ are simply assumed to be red upon accretion. Second, some of the stripped galaxies will not yet have transitioned to the red sequence because they will not yet have consumed their inner gas disk. Setting the "red" fraction to half of the "stripped" fraction provides a nice by eye fit to the observations, as shown in Figure 4(e). Considering its simplicity, the toy model now matches the observations remarkably well.

Figure 4(f) shows the same toy model for galactic halo stripping. While the predicted $f_{r}$ does increase from the lowest 
mass groups to the two higher $M_{\mathrm{gr}}$ bins, the steady increase with $M_{\mathrm{gr}}$ is not predicted. Varying the parameters of the toy model, for instance by increasing the width of the $M_{\text {eff }}$ distribution, does little to alleviate this discrepancy. Similarly, the new toy model predicts a variation with $M_{r}$ only in the lowest mass groups, the opposite of what is observed. Both of these discrepancies reflect the large efficiency of galactic halo stripping in the analytical model. A full SAM with stronger galactic halo stripping should be run to test the toy model. It is known that current SAMs with less efficient partial stripping reproduce observations, but it is not known what constraints the SAMs place on the efficiency of stripping. One should keep in mind that the current analytical model may still underestimate the efficiency of galactic halo stripping. Successfully observing galactic halos around disk galaxies will allow the model to be correctly normalized as well as place important new constraints on SAMs. At a predicted bolometric X-ray luminosity of $10^{39} \mathrm{erg} \mathrm{s}^{-1}$ for an MW-like galaxy, the analytical toy model predicts no dependence of $f_{r}$ on $M_{\mathrm{gr}}$.

When the bottom and top panels of Figure 4 are compared, the need to consider the distribution in $M_{\text {eff }}$ rather than the typical stripping efficiency alone becomes clear. While the correlation between $f_{r}$ and $M_{\mathrm{gr}}$ is expected to be strong as is observed, a significantly weaker correlation is expected between $f_{r}$ and $M_{r}$. The range in $M_{\mathrm{gr}}$ is two orders of magnitude, larger or at least comparable to the width of the $M_{\text {eff }}$ distribution, but the range in luminosity is only one order of magnitude. This plays as large a role in setting the expected strength of the correlation as the underlying variation in the typical RPS efficiency across the range of $M_{r}$.

The results of applying the analytical RPS model to the SDSS groups and constructing a toy model of $f_{r}$ indicate that inflow plus RPS can be invoked to explain the observed correlations between $f_{r}$ and both $M_{\mathrm{gr}}$ and $M_{r}$ in the SDSS groups. To better test for the weak correlation between $f_{r}$ and $M_{r}$ either a larger group sample or a group sample with a wider dynamic range in luminosity should be used.

The toy model may place constraints on the disk inflow plus RPS scenario. First, the model requires that a large fraction of the gas disk must be stripped before the SFR is affected. This is consistent with the rough estimates presented in Section 4.1. Second, the flat relationship between $f_{r}$ and $M_{r}$ seems to favor a wide distribution in $M_{\mathrm{eff}}$ at fixed $M_{r}$ and $M_{\mathrm{gr}}$. A more realistic treatment of both accretion histories and the relation between the stripped gas fraction and the time to transition to the red sequence may effectively increase the width of the distribution. Finally, the relatively low $f_{r}$ in massive groups suggests that roughly half of the satellites were accreted in the last few gigayears, which could be checked with simulations.

There is one discrepancy between the toy model and the observations. While in the toy model the greatest correlation between $f_{r}$ and $M_{r}$ is seen for the intermediate $M_{\mathrm{gr}}$, in the observations the greatest correlation is seen for the high $M_{\mathrm{gr}}$ groups. This should be taken lightly as the toy model does not consider either the effects of hierarchical structure formation or dark matter dynamics within virialized structures.

\section{CONCLUSIONS}

New observational results have been presented demonstrating that a previously observed increase in the red fraction among satellite galaxies with increasing group mass arises from a similar increase among satellite galaxies with disk-like light profiles.
In conjunction with X-ray observations of spiral galaxies and cosmological simulations of disk growth, both of which indicate that spiral galaxies lack a galactic halo that is dense enough to resist complete stripping in groups, these results challenge the hypothesis that the observed correlation between $f_{r}$ and $M_{\mathrm{gr}}$ can be explained by partial stripping of the hot galactic halo. They call for a new theory of star formation fueling for satellite galaxies.

I advance the hypothesis that inflow within the HI disk is capable of supporting star formation in disk satellite galaxies at close to its normal rate for several gigayears and that the partial stripping of this gas disk is responsible for the correlation between $f_{r}$ and $M_{\mathrm{gr}}$. By increasing the time for which disk satellite galaxies continue to form stars, this hypothesis addresses the excess of observed blue satellites as compared to conventional SAM predictions that incorporate complete galactic halo stripping. This mechanism is functionally similar to partial stripping of the galactic halo, but is consistent with observations of real disk galaxies and with the latest gaseous cosmological simulations.

An analytical model that captures not only the environmental dependence of RPS, but also the great variation in the efficiency of RPS at fixed $M_{\mathrm{gr}}$ and $L_{\mathrm{sat}}$, has been used to place RPS in the context of the SDSS groups and to confirm that the observed correlations are consistent with partial RPS. The SDSS satellite galaxies are in the regime of partial RPS, in which the stripping efficiency increases with $M_{\mathrm{gr}}$. This is consistent with the observed correlation between $f_{r}$ and $M_{\mathrm{gr}}$. In addition, the relatively weak correlation between $f_{r}$ and $M_{r}$, despite a strong correlation between the predicted stripped gas fraction and $M_{r}$, can be understood by comparing the wide variation in stripping efficiency at fixed $M_{\mathrm{gr}}$ and $L_{\mathrm{sat}}$ to the narrow range of $L_{\mathrm{sat}}$ present in the catalog.

This work demonstrates the power of using the light profile, specifically the $i$-band Sérsic index, to distinguish between physical processes on the bases of whether they alter structure, or only color, and whether they operate in disk or elliptical galaxies. As $n_{i}$ is a noisy measurement, and color and structure are correlated, such studies should be approached with care. Potential biases arising from noise in $n_{i}$ were considered and found to have only minor effects on the results.

This work emphasizes the interplay between detailed studies of gas accretion and star formation fueling and the study of the full galaxy population. Studies of galaxy populations can place constraints on models of accretion and star formation. If the handful of massive spiral galaxies that have been observed in the X-ray are typical, then a simple accretion model centered on the hot galactic halo cannot account for the observed colors of satellite galaxies with disk-like light profiles. Such a simple model is not particularly well informed by either X-ray observations or the most recent models of disk formation and evolution. It is not surprising that this model is incorrect, but it is informative that the study of galaxy populations can show it is incorrect.

The results presented here warrant the adoption of a more complex model of gas accretion when modeling the evolution of the galaxy population and point toward disk galaxies as a prime candidate for improved modeling. The new model should begin by incorporating cold mode gas accretion and should produce spiral galaxies with realistic galactic halos. The current work suggests that such a model would require an improved treatment of gas dynamics within the cold disk in order to reproduce observations. 
This project was advised by D. N. Spergel and funded by NASA Grant Award NNG04GK55G and NSF Grant 0707731. The author thanks A. Berlind, M. Blanton, and D. Hogg for the use of the SDSS group catalog and for useful discussions. The author also thanks the anonymous referees for useful comments and critiques.

Funding for the SDSS has been provided by the Alfred P. Sloan Foundation, the Participating Institutions, the National Aeronautics and Space Administration, the National Science Foundation, the U.S. Department of Energy, the Japanese Monbukagakusho, and the Max Planck Society. The SDSS Web site is http://www.sdss.org/.

The SDSS is managed by the Astrophysical Research Consortium (ARC) for the Participating Institutions. The Participating Institutions are The University of Chicago, Fermilab, the Institute for Advanced Study, the Japan Participation Group, The Johns Hopkins University, the Korean Scientist Group, Los Alamos National Laboratory, the Max-Planck-Institute for Astronomy (MPIA), the Max-Planck-Institute for Astrophysics (MPA), New Mexico State University, University of Pittsburgh, University of Portsmouth, Princeton University, the United States Naval Observatory, and the University of Washington.

\section{REFERENCES}

Abadi, M. G., Moore, B., \& Bower, R. G. 1999, MNRAS, 308, 947

Baldry, I. K., Glazebrook, K., Brinkman, J., Ivezić, Ž., Lupton, R. H., Nichol, R. C., \& Szalay, A. S. 2004, ApJ, 600, 681

Baldry, I. K., Glazebrook, K., \& Driver, S. P. 2008, MNRAS, 388, 945

Balogh, M. L., Navarro, J. F., \& Morris, S. L. 2000, ApJ, 540, 113

Balogh, M. L., Baldry, I. K., Nichol, R., Miller, C., Bower, R., \& Glazebrook, K. 2004, ApJ, 615, L101

Bekki, K., Couch, W. J., \& Shioya, Y. 2002, ApJ, 577, 651

Bell, E. F. 2002, ApJ, 581, 1013

Benson, A. J., Bower, R. B., Frenk, C. S., \& White, S. D. M. 2000, MNRAS, 314, 557

Berlind, A. A., et al. 2006, ApJS, 167, 1

Bigiel, F., Leroy, A., Walter, F., Brinks, E., de Block, W. J. G., Madore, B., \& Thornley, M. D. 2008, AJ, 136, 2846

Birnboim, Y., \& Dekel, A. 2003, MNRAS, 345, 349

Blanton, M. R., Lin, H., Lupton, R. H., Maley, F. M., Young, N., Zehavi, I., \& Loveday, J. 2003a, AJ, 125, 2276

Blanton, M. R., et al. 2003b, ApJ, 594, 186

Blanton, M. R., et al. 2003c, AJ, 125, 2348

Blanton, M. R., et al. 2003d, ApJ, 592, 819

Blanton, M. R., Berlind, A. A., \& Hogg, D. W. 2007, ApJ, 664, 791

Blanton, M. R., Eisenstein, D., Hogg, D. W., Schlegel, D. J., \& Brinkmann, J. 2005a, ApJ, 629, 143B

Blanton, M. R., Eisenstein, D. J., Hogg, D. W., \& Zehavi, I. 2006b, ApJ, 645, 977

Blanton, M. R., et al. 2005c, AJ, 129, 2562

Bower, R. G., Benson, A. J., Malbon, R., Helly, J. C., Frenk, C. S., Baugh, C. M., Cole, S., \& Lacey, C. G. 2006, MNRAS, 370, 645

Boylan-Kilchin, M., Ma, C.-P., \& Quataert, E. 2008, MNRAS, 383, 93

Bravo-Alfaro, H., Cayatte, V., van Gorkom, J., \& Balkowski, C. 2000, AJ, 119, 580

Cayatte, V., Kotanyi, C., Balkowski, C., \& van Gorkom, J. H. 1994, AJ, 107, $1003 \mathrm{C}$

Chung, A., van Gorkom, J. H., Kenney, J. D. P., \& Vollmer, B. 2007, ApJ, 659, L115

Collister, A. A., \& Lahav, O. 2005, MNRAS, 361, 415

Combes, F. 2007, in IAU Symp. 245, Formation and Evolution of Galaxy Bulges, ed. M. Bureau, E. Athanssoula, \& B. Barbuy (Cambridge: Cambridge Univ. Press), 151

Cooper, M. C., et al. 2006, MNRAS, 370, 198

Cooper, M. C., et al. 2007, MNRAS, 376, 1445

Croton, D. J., et al. 2005, MNRAS, 356, 1155

Croton, D. J., et al. 2006, MNRAS, 365, 11

Dekel, A., \& Birnbiom, Y. 2006, MNRAS, 368, 2

Dressler, A. 1980, ApJ, 236, 351

Eisenstein, D. J., et al. 2001, AJ, 122, 2267

Font, A. S., et al. 2008, MNRAS, 389, 1619
Fukugita, M., Ichikawa, T., Gunn, J. E., Doi, M., Shimasaku, K., \& Schneider, D. P. 1996, AJ, 111, 1748

Gerke, B. F., et al. 2007, MNRAS, 376, 1425

Gill, S. P. D., Knebe, A., Gibson, B. K., \& Dopita, M. A. 2004, MNRAS, 351, 410

Giovanelli, R., \& Haynes, M. P. 1983, AJ, 88, 881

Gomez, P. L., et al. 2003, ApJ, 584, 210

Goto, T., Yamauchi, C., Fujita, Y., Okamura, S., Sekiguchi, M., Smail, I., Bernardi, M., \& Gomez, P. L. 2003, MNRAS, 346, 601

Göttlober, S., Klypin, A., \& Kravtsov, A. V. 2001, ApJ, 546, 223

Gunn, J. E., \& Gott, J. R. 1972, ApJ, 176, 1

Helsdon, S. F., \& Ponman, T. J. 2000, MNRAS, 315, 356

Hester, J. A. 2006a, ApJ, 647, 910

Hester, J. A. 2006b, astro-ph/0610089

Hogg, D. W., Finkbeiner, D. P., Schlegel, D. J., \& Gunn, J. E. 2001, AJ, 122, 2129

Hogg, D. W., et al. 2003, ApJ, 585, L5

Ivezić, Ž., et al. 2004, Astron. Nachr., 325, 583

Kang, X., \& van den Bosch, F. C. 2008, ApJ, 676, L101

Kauffmann, G., White, S. D. M., Heckman, T. M., Menard, B., Brinchmann, J., Charlot, S., Tremonti, C., \& Brinkmann, J. 2004, MNRAS, 353, 713

Kauffmann, G., et al. 2003a, MNRAS, 341, 33

Kauffmann, G., et al. 2003b, MNRAS, 341, 54

Kelm, B., Focardi, P., \& Sorrnetino, G. 2005, A\&A, 442, 117

Kenney, J. D. P., Crowl, H., van Gorkom, J., \& Vollmer, B. 2004a, in IAU Symp. 217, Recycling Intergalactic and Interstellar Matter, ed. P.-A. Duc, J. Braine, \& E. Brinks (San Francisco, CA: ASP), 370

Kenney, J. D. P., \& Koopmann, R. A. 1998, AJ, 117, 181K

Kenney, J. D. P., van Gorkom, J., \& Vollmer, B. 2004b, AJ, 127, 3361K

Kennicutt, R. C. 1998, ApJ, 498, 541

Kereš, D., \& Hernquist, L. 2009, ApJ, 700, L1

Kereš, D., Katz, N., Weinberg, D. H., \& Romeel, D. 2005, MNRAS, 363, 2

Knebe, A., Power, C., Stuart, P. D. G., \& Gibson, B. K. 2006, MNRAS, 368, 741

Larson, R. B., Tinsley, B. M., \& Cladwell, C. N. 1980, ApJ, 237, 692

Li, C., Kauffmann, G., Jing, Y. P., White, S. D. M., Borner, G., \& Cheng, F. Z. 2006, MNRAS, 368, 37

Lin, D. N. C., \& Pringle, J. E. 1987a, ApJ, 320, L87

Lin, D. N. C., \& Pringle, J. E. 1987b, MNRAS, 225, 607

Lupton, R. H., Gunn, J. E., Ivezić, Ž, Knapp, G. R., Kent, S., \& Yasuda, N. 2001 , in ASP Conf. Ser. 238, Astronomical Data Analysis Software and Systems X, ed. F. R. Harnden, Jr., F. A. Primini, \& H. E. Payne (San Francisco, CA: ASP), 269

Madgwick, et al. 2002, MNRAS, 333, 133

Marcolini, A., Brighenti, F., \& D'Ercole, A. 2003, MNRAS, 345, 1329

Martin, C. L., \& Kenniuctt, R. C. 2001, ApJ, 555, 301

Martínez, H. J., Zandivarez, A., Domínguez, M., Merchán, M. E., \& Lambas, D. G. 2002, MNRAS, 333, L31

McCarthy, I. G., Frenk, C. S., Font, A. S., Lacey, C. G., Bower, R. G., Mitchell, N. L., Balogh, M. L., \& Theuns, T. 2008, MNRAS, 383, 593

McNally, C. P., Wadsley, J., \& Couchman, H. M. P. 2009, ApJ, 697, L162

Mohr, J. J., Mathiesen, B., \& Evrard, A. E. 1999, ApJ, 517, 627

Moore, B., Lake, G., \& Katz, N. 1998, ApJ, 495, 139

Norberg, P., et al. 2002, MNRAS, 332, 827

Olivier, S. S., Blumenthal, G. R., \& Primack, J. R. 1991, MNRAS, 252, 102

Osmond, J. P. F., \& Ponman, T. J. 2004, MNRAS, 350, 1511

Pier, J. R., Munn, J. A., Hindsley, R. B., Hennessy, G. S., Kent, S. M., Lupton, R. H., \& Ivezić, Ž. 2003, AJ, 125, 1559

Quilis, V., Moore, B., \& Bower, R. 2000, Science, 288, 1617

Quintero, A. D., Berlind, A. A., Blanton, M. R., \& Hogg, D. W. 2006, astro-ph/0611361

Rasmussen, J., Sommer-Larsen, J., Pedersen, K., Toft, S., Benson, A., Bower, R. B., \& Grove, L. F. 2009, ApJ, 697, 79

Richards, G. T., et al. 2002, AJ, 123, 2945

Roediger, E., \& Brüggen, M. 2007, MNRAS, 380, 1399

Roediger, E., \& Hensler, G. 2005, A\&A, 433, 875

Salim, S., \& Rich, R. M. 2010, ApJ, 714, L290

Sanderson, A. J. R., \& Ponman, T. J. 2003, in Clusters of Galaxies: Probes of Cosmological Structure and Galaxy Evolution, ed. J. S. Mulchaey, A. Dressler, \& A. Oemler (Pasadena, CA: Carnegie Observatories)

Schlegel, D. J., Finkbeiner, D. P., \& Davis, M. 1998, ApJ, 500, 525

Schulz, S., \& Struck, C. 2001, MNRAS, 328, 185

Shu, F. 1992, Gas Dynamics, Vol. 2 (Sausalito, CA: Univ. Science Books)

Smith, J. A., et al. 2002, AJ, 123, 2121 
Solanes, J. M., Manrique, A., Garcia-Gomez, C., Gonzalez-Casado, G., Giovanalli, R., \& Haynes, M. P. 2001, ApJ, 548, 97

Strateva, I., et al. 2001, AJ, 122, 1861

Strauss, M. A., et al. 2002, AJ, 124, 1810

Sun, M., Jones, C., Forman, W., Vikhlilin, A., Donahue, M., \& Voit, M. 2007, ApJ, 657, 197

Swaters, R. A., van Albada, T. S., van der Hulst, J. M., \& Sancisi, R. 2002, A\&A, 390,829

Tanaka, M., Goto, T., Okamaru, S., Shimasaku, K., \& Brunkmann, J. 2004, AJ, 128,2677

Tasitsiomi, A., Kravtsov, A. V., Wechsler, R. H., \& Primack, J. R. 2004, ApJ, 614,533

Thilker, D. A., et al. 2007, ApJS, 173, 538 van den Bosch, F. C., Aquino, D., Yang, X., Mo, H. J., Pasquali, A., McIntosh, D. H., Weinmann, S. M., \& Kang, X. 2008, MNRAS, 387, 79

Weinmann, S. M., van den Bosch, F. C., Yang, X., \& Mo, H. J. 2006a, MNRAS, 366,2

Weinmann, S. M., van den Bosch, F. C., Yang, X., Mo, H. J., Croton, D. J., \& Moore, B. 2006b, MNRAS, 372, 1161

Wyder, T. K., et al. 2009, ApJ, 696, 1834

Yang, X., Mo, H. J., Jing, Y. P., \& van den Bosch, F. C. 2005, MNRAS, 358 217

Yang, X., Mo, H. J., \& van den Bosch, F. C. 2003, MNRAS, 339, 1057

York, D. G., et al. 2000, AJ, 120, 1579

Zheng, Z., et al. 2005, ApJ, 633, 791 